\title{
A study of one-dimensional transport of Bose-Einstein condensates using exterior complex scaling
}

\author{
Julien Dujardin · Alejandro Saenz • Peter Schlagheck
}

Received: date / Revised version: date

Keywords guided atom lasers, Bose-Einstein condensates, Gross-Pitaevskii equation, open Bose-Hubbard system, smooth exterior complex scaling, transparent boundary conditions.

PACS 02.60.Lj,67.85.Hj,67.85.De,02.60.Cb

Abstract We numerically investigate the one-dimensional transport of Bose-Einstein condensates in the context of guided atom lasers using a mean-field description of the condensate in terms of a spatially discretized Gross-Pitaevskii equation. We specifically consider a waveguide configuration in which spatial inhomogeneities and nonvanishing atom-atom interactions are restricted to a spatially localized scattering region of finite extent. We show how the method of smooth exterior complex scaling can be implemented for this particular configuration in order to efficiently absorb the outgoing flux within the waveguide. A numerical comparison with the introduction of a complex absorbing potential as well as with the analytically exact elimination of the dynamics of the free non-interacting motion outside the scattering region, giving rise to transparent boundary conditions, clearly confirms the accuracy and efficiency of the smooth exterior complex scaling method.

\section{Introduction}

The perspective to realize atomtronic devices [1-3] as well as the exploration of transport features that are

Julien Dujardin and Peter Schlagheck

Département de Physique, Université de Liège, 4000 Liège, Belgium

Alejandro Saenz

Institut für Physik, Humboldt-Universität zu Berlin, 12489

Berlin, Germany known from electronic mesoscopic systems 4 have strongly stimulated the research on the dynamical properties of ultracold atoms in open systems. While fermionic atoms provide direct analogies with the electronic case [4 6], the use of a bosonic atomic species brings along new aspects and challenges for the atomic transport problem [7, 8. It is in this context particularly relevant to quantitatively understand the conceptual link between a mesoscopic Bose-Einstein condensate, which may serve as a reservoir for a bosonic transport setting, and the microscopic dynamics of an ensemble of few interacting atoms that encounter each other e.g. within a transistor-like device. A particularly promising configuration for the experimental study of these latter aspects is provided by the guided atom laser 9 11 in which atoms are coherently outcoupled from a trapped Bose-Einstein condensate into an optical waveguide. A coherent atomic beam can thereby be created and injected onto engineered optical scattering geometries, which would allow one to study bosonic many-body scattering at well-defined incident energy.

A theoretical modelling of such waveguide scattering processes within guided atom lasers faces the problem of dealing with an open system in a many-body context. Within the framework of the mean-field approximation described by the nonlinear Gross-Pitaevskii equation, this problem can be solved to a satisfactory degree by imposing absorbing boundary conditions 12 at the two open ends of the numerical grid representing the waveguide, which are suitably defined in order to match the dispersion relation of the expected outgoing waves 13, 14. While this approach provides a reasonably efficient absorption of outgoing Gross-Pitaevskii waves even in the presence of dynamical instabilities [15], it ultimately breaks down if quantum fluctuations beyond the Gross-Pitaevskii approximation are taken into ac- 
count in the theoretical description of the bosonic scattering process [16]. Complex absorbing potentials (CAPs) [17,18, that exhibit a nonvanishing imaginary part can still be introduced in that case in order to damp the outgoing flux. However, their numerical implementation requires great care in order to suppress unwanted backreflections of outgoing waves at the onset of the artificially introduced imaginary potential (see Refs. [19,20 for successful applications of CAPs in the context of the Gross-Pitaevskii equation).

The method of Complex Scaling (CS) [21+27] provides a more satisfactory alternative from a conceptual point of view. This method essentially consists (in 1D) in the rotation $x \mapsto z=x \exp (i \theta)$ of the spatial coordinate in the complex plane by a suitably chosen angle $\theta>0$. Decaying quasi-bound states that exhibit outgoing boundary conditions become square integrable by this transformation and can thereby be computed in an open system. The complex scaling approach can formally be generalized to the nonlinear Gross-Pitaevskii equation 28,29. However, its practical implementation in this latter context poses substantial numerical difficulties concerning the proper evaluation of the nonlinear term in the complex rotated frame [29 31].

In this paper, we focus on the method of Exterior Complex Scaling (ECS) 32] which is particularly suited for open systems in which potential scattering and (meanfield) particle-particle interaction effects are restricted to a finite spatial region. This method consists in a complex rotation of the position coordinate applied only to the asymptotic spatial domain of freely outgoing and noninteracting particles. ECS has been applied in a wide range of problems such as computing the probability distribution of excitations to the electronic continuum of $\mathrm{HeT}^{+}$following the $\beta$ decay of the $\mathrm{T}_{2}$ molecule 33, molecular photoionization 34, electron-hydrogen collisions [35, 36] and also strong-field infrared photoionization of atoms [37-40]. While this approach exactly reproduces the true decay behaviour in the open quantum system from a formal point of view (in contrast to the introduction of CAPs), numerical imprecisions are necessarily introduced through the discretization of space in the finite-difference approximation [36] of the Gross-Pitaevskii approximation. This problem can be overcome by using high rank finite elements [41, 42] or a B-spline basis [43] instead of a finite-difference representation. Alternatively, an analytic transition function can be used to interpolate from the scaled "outer" domain to the unscaled "inner" domain which may contain all sorts of nontrivial scattering and interaction phenomena. This latter method is named Smooth Exterior Complex Scaling (SECS) 44]. It has been used, for example, to compute doubly excited states of the helium atom [45] and to investigate the dynamical stability of stationary scattering states of a Bose-Einstein condensate in two-dimensional billiard geometries [46].

The main aim of this study is to assess the applicability of smooth exterior complex scaling to the mean-field transport of Bose-Einstein condensates in one-dimensional waveguides using the finite-difference approximation. We therefore represent, as described in Sec. 2. the waveguide by means of a discrete one-dimensional chain which is at some point connected to a separate site representing the reservoir trap of the atom laser. Scattering and interaction phenomena are assumed to be restricted to a finite spatial domain within this chain. As is shown in Sec. 3, this crucial assumption allows us to formally separate this central domain from the two attached semi-infinite "leads" featuring free non-interacting motion. This gives rise to perfectly transparent boundary conditions which render, however, the numerical propagation of the system rather time-consuming. In Sec. 4, smooth exterior complex scaling is then introduced to this open system as a feasible alternative. Finally, numerical results comparing the use of smooth exterior complex scaling, of complex absorbing potentials, as well as of the transparent boundary conditions derived in Sec. 3 are presented in Sec. 5 .

\section{1D Bose-Hubbard chain with a source}

We consider an infinite one-dimensional (1D) Bose-Hubbard $(\mathrm{BH})$ system representing the transverse ground mode of a 1D waveguide in a finite-difference representation. This BH chain is connected at one of its sites to one additional site representing a reservoir of BoseEinstein condensed atoms with the chemical potential $\mu$, as illustrated in Fig. 1. The many-body Hamiltonian of this system reads

$$
\begin{aligned}
\hat{\mathcal{H}}=\sum_{\ell=-\infty}^{+\infty} & {\left[-J\left(\hat{a}_{\ell+1}^{\dagger} \hat{a}_{\ell}+\hat{a}_{\ell}^{\dagger} \hat{a}_{\ell+1}\right)\right.} \\
& \left.+\frac{g_{\ell}}{2} \hat{n}_{\ell}\left(\hat{n}_{\ell}-1\right)+V_{\ell} \hat{n}_{\ell}\right] \\
& +\kappa^{*}(t) \hat{b}^{\dagger} \hat{a}_{\ell_{S}}+\kappa(t) \hat{a}_{\ell_{S}}^{\dagger} \hat{b}+\mu \hat{b}^{\dagger} \hat{b},
\end{aligned}
$$

where we define by $\hat{a}_{\ell}$ and $\hat{a}_{\ell}^{\dagger}$ the annihilation and creation operators, respectively, on the site $\ell$ of the chain, with $\hat{n}_{\ell}=\hat{a}_{\ell}^{\dagger} \hat{a}_{\ell}$ the corresponding number operator, and by $\hat{b}$ and $\hat{b}^{\dagger}$ the annihilation and creation operator of the reservoir to which the chain is connected at the site $\ell_{S}$. The hopping strength $J$, the on-site interaction strength $g_{\ell}$, as well as the on-site potential $V_{\ell}$ can be determined from the Hamiltonian of the underlying continous system through the discretization of the spatial 
coordinate along the waveguide. The coupling strength $\kappa(t)$, on the other hand, is related to the outcoupling process of atoms from the reservoir and can be controlled in a time-dependent manner (e.g. through the variation of the intensity of a radiofrequency field in the case of Refs. 9, 47]). We should mention, however, that the framework developed here does not exclusively apply to guided atom lasers, but could also be used in the context of analogous transport processes taking place within optical lattices.

In the Heisenberg representation, the time evolution of the annihilation operators $\hat{a} \equiv \hat{a}_{\ell}(t)$ and $\hat{b} \equiv \hat{b}(t)$ is given by the Heisenberg equations (we set $\hbar=1$ in the following)

$$
\begin{aligned}
i \frac{\partial \hat{a}_{\ell}(t)}{\partial t}= & V_{\ell} \hat{a}_{\ell}(t)-J\left[\hat{a}_{\ell-1}(t)+\hat{a}_{\ell+1}(t)\right] \\
& +g_{\ell} \hat{a}_{\ell}^{\dagger}(t) \hat{a}_{\ell}(t) \psi_{\ell}(t)+\kappa(t) \delta_{\ell, \ell_{S}} \hat{b}(t) \\
i \frac{\partial \hat{b}(t)}{\partial t}= & \mu \hat{b}(t)+\kappa^{*}(t) \hat{a}_{\ell_{S}}(t) .
\end{aligned}
$$

In accordance with the working principle of an atom laser, we consider an initial state at time $t_{0}$ in which the source is populated with a very large number $N \rightarrow \infty$ of atoms and the chain is empty. Moreover, we consider a very weak coupling strength $\kappa(t) \rightarrow 0$ which tends to zero such that $N|\kappa(t)|^{2}$ remains finite. This combined limit gives rise to a finite population within the chain, which is (at time-independent $\kappa$ ) alimented by a steady flux of atoms from the reservoir.

In the following, we consider the classical mean-field regime of large on-site densities and weak interaction strengths within the Bose-Hubbard chain, in which (at finite evolution time $t$ ) the system can be described using c-numbers instead of operators. The dynamics of the system in this regime is described by the nonlinear Gross-Pitaevskii (GP) equation

$$
\begin{aligned}
i \frac{\partial \psi_{\ell}(t)}{\partial t} & =\left(V_{\ell}-\mu\right) \psi_{\ell}(t)-J\left(\psi_{\ell+1}(t)+\psi_{\ell-1}(t)\right) \\
& +g_{\ell}\left|\psi_{\ell}(t)\right|^{2} \psi_{\ell}(t)+\delta_{\ell, \ell_{S}} \kappa(t) \chi(t) \\
i \frac{\partial \chi(t)}{\partial t} & =\kappa^{*}(t) \psi_{\ell_{S}}(t)
\end{aligned}
$$

where we define the amplitudes $\psi_{\ell}(t)=\left\langle\hat{a}_{\ell}(t)\right\rangle e^{-i \mu t}$ and $\chi(t)=\langle\hat{b}(t)\rangle e^{-i \mu t}$ with the initial conditions $\psi_{\ell}\left(t_{0}\right)=$ 0 and $\chi\left(t_{0}\right)=\sqrt{N}$. From Eqs. (3a) and (3b) we can infer that $\chi(t)=\sqrt{\mathcal{N}}\left(1+\mathcal{O}\left(|\kappa|^{2}\right)\right.$ at finite $t-t_{0}$, and as a consequence we can neglect the time dependence of $\chi$ in the limit $\kappa \rightarrow 0$. We then obtain a discrete nonlinear Schrödinger equation with a source term:

$$
\begin{aligned}
i \frac{\partial \psi_{\ell}(t)}{\partial t}= & \left(V_{\ell}-\mu\right) \psi_{\ell}(t) \\
& -J\left(\psi_{\ell+1}(t)+\psi_{\ell-1}(t)\right) \\
& +g_{\ell}\left|\psi_{\ell}(t)\right|^{2} \psi_{\ell}(t)+\delta_{\ell, \ell_{S}} \kappa(t) \sqrt{\mathcal{N}} .
\end{aligned}
$$

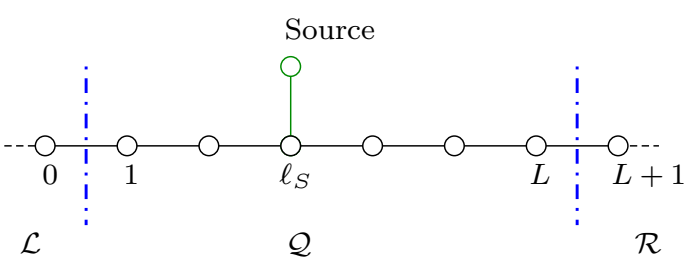

Fig. 1 (color online) One-dimensional infinite BH system with an additional site for the source. The zone $\mathcal{Q}$ is defined between the dot-dashed lines.

\section{Transparent boundary conditions}

The standard procedure for a numerical study of the time-dependent dynamics within an infinite chain consists in defining a sufficiantly large "simulation box" containing a finite number of sites. The choice of the boundary conditions at the edges of the box is, in general, irrelevant for wave packet evolution processes that evolve within a finite time; it does, however, matter for the type of scattering processes that we consider here: choosing hard-wall or periodic boundary conditions would rather quickly lead to unwanted reflections of the matter wave at the artificially introduced boundaries of the box.

To avoid such artificial backreflections, we can introduce transparent boundary conditions (TBC), making use of the fact, as explained in the introduction, that the scattering potential and the interaction strength are non-zero only in a finite region of space. To this end, we formally divide the system in three parts, namely the semi-infinite left and right parts $\mathcal{L}$ and $\mathcal{R}$ where neither interaction nor scattering takes place, and the finite central part $\mathcal{Q}$ consisting of $L$ sites numbered from 1 to $L$, which contains potential scattering, atom-atom interaction, as well as the link to the source (see Fig. 1). For the sake of compactness of the formalism, we regroup all the amplitudes $\psi_{\ell}$ into a state $|\psi\rangle$ defined through

$|\psi\rangle=\sum_{\ell=-\infty}^{+\infty} \psi_{\ell}|\ell\rangle$,

where the on-site states $|\ell\rangle$ form an orthonormal basis $\left\langle\ell \mid \ell^{\prime}\right\rangle=\delta_{\ell, \ell^{\prime}}$. Formally Eq. (4) can then be expressed as

$i \frac{\partial|\psi\rangle}{\partial t}=\mathcal{H}|\psi\rangle=\left[\mathcal{H}_{f}+\mathcal{V}+\mathcal{U}(\psi)\right]|\psi\rangle+|S\rangle$

with $|S\rangle=\kappa(t) \sqrt{\mathcal{N}}\left|\ell_{0}\right\rangle$, where we decompose the GrossPitaveksii Hamiltonian $\mathcal{H}$ in the free motion on the chain described by $\mathcal{H}_{f}$, the scattering potential included in $\mathcal{V}$, and the nonlinear interaction term $\mathcal{U}(\psi)$. The cor- 
responding matrix elements in the local basis are

$$
\begin{aligned}
\left\langle\ell\left|\mathcal{H}_{f}\right| \ell^{\prime}\right\rangle & =-\mu \delta_{\ell, \ell^{\prime}}-J\left(\delta_{\ell, \ell^{\prime}-1}+\delta_{\ell, \ell^{\prime}+1}\right), \\
\left\langle\ell|\mathcal{V}| \ell^{\prime}\right\rangle & =\delta_{\ell, \ell^{\prime}} V_{\ell} \\
\left\langle\ell|\mathcal{U}(\psi)| \ell^{\prime}\right\rangle & =\delta_{\ell, \ell^{\prime}} g_{\ell}\left|\psi_{\ell}\right|^{2}
\end{aligned}
$$

We can now define the division of the system described above using the Feshbach projection formalism with the three projectors

$$
\begin{aligned}
& P_{\mathcal{L}}=\sum_{\ell=-\infty}^{0}|\ell\rangle\langle\ell|, \\
& P_{\mathcal{Q}}=\sum_{\ell=1}^{L}|\ell\rangle\langle\ell|, \\
& P_{\mathcal{R}}=\sum_{\ell=L+1}^{\infty}|\ell\rangle\langle\ell| .
\end{aligned}
$$

This gives rise to the three coupled evolution equations

$$
\begin{aligned}
i \frac{\partial\left|\psi^{(\mathcal{L})}\right\rangle}{\partial t} & =\mathcal{H}_{\mathcal{L}}\left|\psi^{(\mathcal{L})}\right\rangle+\mathcal{W}_{\mathcal{L} \mathcal{Q}}\left|\psi^{(\mathcal{Q})}\right\rangle, \\
i \frac{\partial\left|\psi^{(\mathcal{Q})}\right\rangle}{\partial t} & =\left(\mathcal{H}_{\mathcal{Q}}+\mathcal{V}_{\mathcal{Q}}+\mathcal{U}_{\mathcal{Q}}(\psi)\right)\left|\psi^{(\mathcal{Q})}\right\rangle+|S\rangle \\
& +\mathcal{W}_{\mathcal{Q} \mathcal{L}}\left|\psi^{(\mathcal{L})}\right\rangle+\mathcal{W}_{\mathcal{Q R}}\left|\psi^{(\mathcal{R})}\right\rangle, \\
i \frac{\partial\left|\psi^{(\mathcal{R})}\right\rangle}{\partial t} & =\mathcal{H}_{\mathcal{R}}\left|\psi^{(\mathcal{R})}\right\rangle+\mathcal{W}_{\mathcal{R} \mathcal{Q}}\left|\psi^{(\mathcal{Q})}\right\rangle
\end{aligned}
$$

where we define $\left|\psi^{(\mathcal{X})}\right\rangle=P_{\mathcal{X}}|\psi\rangle, \mathcal{H}_{\mathcal{X}}=P_{\mathcal{X}} \mathcal{H}_{f} P_{\mathcal{X}}$, with $\mathcal{X}, \mathcal{Y}$ being equal to $\mathcal{Q}, \mathcal{L}$ or $\mathcal{R}$, as well as

$$
\begin{aligned}
& \mathcal{W}_{\mathcal{L} \mathcal{Q}}=P_{\mathcal{L}} \mathcal{H}_{f} P_{\mathcal{Q}}=-J|0\rangle\langle 1|=\mathcal{W}_{\mathcal{Q} \mathcal{L}}^{\dagger}, \\
& \mathcal{W}_{\mathcal{R} \mathcal{Q}}=P_{\mathcal{R}} \mathcal{H}_{f} P_{\mathcal{Q}}=-J|L+1\rangle\langle L|=\mathcal{W}_{\mathcal{Q} \mathcal{R}}^{\dagger} .
\end{aligned}
$$

The evolution equations (9a), (9c) for the left and the right part are linear and describe a free propagation in a semi-infinite lead. As a consequence, we can formally integrate them and plug the result in the evolution equation (9b) for the central part. This yields

$$
\begin{aligned}
i \frac{\partial\left|\psi^{(\mathcal{Q})}\right\rangle}{\partial t}= & {\left[\mathcal{H}_{\mathcal{Q}}+\mathcal{V}_{\mathcal{Q}}+\mathcal{U}_{\mathcal{Q}}(\psi)\right]\left|\psi^{(\mathcal{Q})}\right\rangle } \\
& -i \int_{t_{0}}^{t} d t^{\prime} \mathcal{W}_{\mathcal{Q} \mathcal{L}} e^{-i\left(t-t^{\prime}\right) \mathcal{H}_{\mathcal{L}}} \mathcal{W}_{\mathcal{L} \mathcal{Q}}\left|\psi^{(\mathcal{Q})}\left(t^{\prime}\right)\right\rangle \\
& -i \int_{t_{0}}^{t} d t^{\prime} \mathcal{W}_{\mathcal{Q} \mathcal{R}} e^{-i\left(t-t^{\prime}\right) \mathcal{H}_{\mathcal{R}}} \mathcal{W}_{\mathcal{R} \mathcal{Q}}\left|\psi^{(\mathcal{Q})}\left(t^{\prime}\right)\right\rangle \\
& +\mathcal{W}_{\mathcal{Q} \mathcal{R}} e^{-i\left(t-t_{0}\right) \mathcal{H}_{\mathcal{R}}}\left|\psi^{(\mathcal{R})}\left(t_{0}\right)\right\rangle \\
& +\mathcal{W}_{\mathcal{Q} \mathcal{L}} e^{-i\left(t-t_{0}\right) \mathcal{H}_{\mathcal{L}}}\left|\psi^{(\mathcal{L})}\left(t_{0}\right)\right\rangle,
\end{aligned}
$$

where the second and third lines describe the decay into the leads and the fourth and fifth lines describe the propagation of the initial conditions within the lead into the scattering region $\mathcal{Q}$.
The integrals in Eq. (12) are calculated using the normalized continuum eigenstates $\left|k^{(\mathcal{L} / \mathcal{R})}\right\rangle$ of the leads, which in the local basis $|\ell\rangle$ can be written as

$\left\langle\ell \mid k^{(\mathcal{L})}\right\rangle=\sqrt{\frac{2}{\pi}} \sin [(\ell-1) k] \quad$ with $\ell<1$

for the left lead and

$\left\langle\ell \mid k^{(\mathcal{R})}\right\rangle=\sqrt{\frac{2}{\pi}} \sin [(\ell-L) k] \quad$ with $\ell>L$

for the right lead, with $0 \leq k \leq \pi,\left\langle k^{(\mathcal{L} / \mathcal{R})} \mid \tilde{k}^{(\mathcal{L} / \mathcal{R})}\right\rangle=$ $\delta(k-\tilde{k})$ and the associated eigenvalues

$E_{k}=-2 J \cos (k)-\mu$.

For the term $\mathcal{W}_{\mathcal{Q} \mathcal{L}} e^{-i \tau \mathcal{H}_{\mathcal{L}}} \mathcal{W}_{\mathcal{L} \mathcal{Q}}$ for instance, we obtain the expression

$$
\left.\mathcal{W}_{\mathcal{Q} \mathcal{L}} e^{-i \tau \mathcal{H}_{\mathcal{L}}} \mathcal{W}_{\mathcal{L} \mathcal{Q}}=J^{2} \int_{0}^{\pi} d k\left|\langle 0| k^{(\mathcal{L}}\right)\right\rangle\left.\right|^{2} e^{-i \tau E_{k}}|1\rangle\langle 1|
$$

which is related to Bessel integrals. This finally yields a finite set of $L$ integro-differential equations

$$
\begin{aligned}
i \frac{\partial \psi_{\ell}}{\partial t}= & \left(V_{\ell}-\mu\right) \psi_{\ell}-J\left(\psi_{\ell-1} \theta_{\ell-1,1}+\psi_{\ell+1} \theta_{L, \ell+1}\right) \\
& +g_{\ell}\left|\psi_{\ell}\right|^{2} \psi_{\ell}+\kappa(t) \sqrt{N} \delta_{\ell, \ell_{S}} \\
& -2 i\left(\delta_{\ell, 1}+\delta_{\ell, L}\right) J^{2} \int_{t_{0}}^{t} d t^{\prime} \mathcal{M}_{1}\left(t-t^{\prime}\right) \psi_{\ell}\left(t^{\prime}\right) \\
& +2 J \delta_{\ell, 1} \sum_{\ell^{\prime}=-\infty}^{0} \mathcal{M}_{\ell^{\prime}-1}\left(t-t_{0}\right) \psi_{\ell^{\prime}}\left(t_{0}\right) \\
& -2 J \delta_{\ell, L} \sum_{\ell^{\prime}=L+1}^{\infty} \mathcal{M}_{\ell^{\prime}-L}\left(t-t_{0}\right) \psi_{\ell^{\prime}}\left(t_{0}\right)
\end{aligned}
$$

with

$\theta_{\ell, \ell^{\prime}}=\left\{\begin{array}{lc}1 & \text { if } \ell \geq \ell^{\prime} \\ 0 & \text { otherwise }\end{array}\right.$

and

$\mathcal{M}_{\ell}(\tau)=\frac{i^{\ell}}{2}\left[J_{\ell-1}(2 J \tau)+J_{\ell+1}(2 J \tau)\right] e^{i \mu \tau}$

where $J_{\ell}(x)$ is the Bessel function of the first kind. As no approximation has been made in this section, Eq. (16) reproduces the true evolution of the infinite system under consideration, described by Eq. (44). The integral term in the third line of Eq. (16) describing the decay into the left and right leads therefore yields a perfectly transparent boundary condition that is defined on the first and last site of the central region. 


\section{Smooth Exterior Complex Scaling}

Within a continuous $1 \mathrm{D}$ system, the method of complex scaling consists in the transformation $x \mapsto z=x e^{i \theta}$ $(x \in \mathbb{R})$ of the position coordinate with $\theta>0$. With this transformation, a stationary wave $\psi(x) \sim e^{i k x}$ becomes $\psi(z) \sim e^{i k z}=e^{i k x \cos \theta} e^{-k x \sin \theta}$ where $k$ is the wavenumber. Waves traveling from left to the right $(k>0)$ are therefore subject to damping for positive $x$, while waves traveling from right to the left $(k<0)$ are damped for negative $x$ (and would be enhanced for positive $x$ [42]). Thus complex scaling allows to describe in a numerically efficient manner the outgoing waves that arise in our 1D scattering problem in which the source is part of the scattering system (see Fig. 1).

For our case, we want to apply the complex scaling transformation to the leads $\mathcal{L}$ and $\mathcal{R}$, while the finite scattering region $\mathcal{Q}$ is supposed to remain unscaled. In order to properly introduce the method of smooth exterior complex scaling (SECS) for this case, we first consider a continuous system with a wavefunction $\psi(x, t)$ that is subject to the Schrödinger equation

$i \frac{\partial}{\partial t} \psi(x, t)=-J \frac{\partial^{2}}{\partial x^{2}} \psi(x, t)$.

We now define a complex analytical function $q(x)$ on the $1 \mathrm{D}$ space and introduce an (in general non-unitary) transformation $\mathcal{U}$ through

$\mathcal{U} \psi(x, t)=\psi(z(x), t)$

where $z(x)$ is defined as

$z(x)=\int_{0}^{x} q\left(x^{\prime}\right) d x^{\prime}$

(assuming, without loss of generality, that the spatial origin $x=0$ is part of $\mathcal{Q}$ ). The evolution of the transformed wavefunction is then given by

$i \frac{\partial \mathcal{U} \psi}{\partial t}(x, t)=-\frac{J}{q^{2}(x)}\left(\frac{\partial^{2}}{\partial x^{2}}-\frac{q^{\prime}(x)}{q(x)} \frac{\partial}{\partial x}\right) \mathcal{U} \psi(x, t)$

where $q^{\prime}(x)$ is the first derivative of $q$ with respect to $x$.

The goal is to choose $q(x)$ such that the Hamiltonian remains unscaled in the $\mathcal{Q}$ region and scaled in the other two regions. For this purpose, we choose $q(x) \rightarrow 1$ within $\mathcal{Q}$ and smoothly $\operatorname{ramp} q(x)$ to $e^{i \theta}$ within the scaled regions. The function $q(x)$ we used in this study reads [4]

$q(x)=1+\left(e^{i \theta}-1\right)\left(1+\frac{f_{+}(x)-f_{-}(x)}{2}\right)$

with

$f_{ \pm}(x)=\tanh \left(\lambda\left(x-x_{ \pm}\right) \pm 2 \pi\right)$ where $\lambda$ is defined as the smoothing parameter and the interval $\left[x_{-}, x_{+}\right]$corresponds to the $\mathcal{Q}$ region.

In order to apply SECS to our BH chain, which can be seen as a discretization of space, we need to define the matrix elements of the spatial derivatives appearing in Eq. (22) within the discrete basis of on-site states $|\ell\rangle$. Within the framework of the finite-difference approximation, we find

$$
\begin{aligned}
\left\langle\ell\left|\frac{\partial}{\partial x}\right| \ell^{\prime}\right\rangle & =\frac{1}{2}\left(\delta_{\ell, \ell^{\prime}+1}-\delta_{\ell, \ell^{\prime}-1}\right), \\
\left\langle\ell\left|\frac{\partial^{2}}{\partial x^{2}}\right| \ell^{\prime}\right\rangle & =\delta_{\ell, \ell^{\prime}+1}+\delta_{\ell, \ell^{\prime}-1}-2 \delta_{\ell, \ell^{\prime}},
\end{aligned}
$$

which yields, using Eq. (7a), the relation

$-J\left\langle\ell\left|\frac{\partial^{2}}{\partial x^{2}}\right| \ell^{\prime}\right\rangle=\left\langle\ell\left|\hat{\mathcal{H}}_{f}\right| \ell^{\prime}\right\rangle+(\mu-2 J) \delta_{\ell, \ell^{\prime}}$

between the free 1D kinetic energy and the hopping term of the $\mathrm{BH}$ model. Defining $\left\langle\ell^{\prime}|q| \ell\right\rangle=q_{\ell} \delta_{\ell, \ell^{\prime}}$, we can discretize Eq (22). Provided that the transition between the scaled and unscaled regions is sufficiently smooth (i.e. $\lambda \ll 1$ ), we can set $q_{\ell-1} \simeq q_{\ell} \simeq q_{\ell+1}$ and the evolution equation (16) now reads

$$
\begin{aligned}
i \frac{\partial \psi_{\ell}}{\partial t}= & \left(V_{\ell}-\mu q_{\ell}\right) \psi_{\ell}+g_{\ell}\left|\psi_{\ell}\right|^{2} \psi_{\ell}+\kappa(t) \sqrt{\mathcal{N}} \delta_{\ell, \ell_{S}} \\
& +2 J\left(q_{\ell}+q_{\ell}^{-1}\right) \psi_{\ell} \\
& -J\left[\frac{1}{q_{\ell+1}}+\frac{1}{2} \frac{q_{\ell+1}^{\prime}}{q_{\ell+1}^{2}}\right] \psi_{\ell+1} \\
& -J\left[\frac{1}{q_{\ell-1}}-\frac{1}{2} \frac{q_{\ell-1}^{\prime}}{q_{\ell-1}^{2}}\right] \psi_{\ell-1} \\
& +2 J \delta_{\ell, 1} \sum_{\ell^{\prime}=-\infty}^{0} \mathcal{M}_{\ell^{\prime}-1}\left(t-t_{0}\right) \psi_{\ell^{\prime}}\left(t_{0}\right) \\
& -2 J \delta_{\ell, L} \sum_{\ell^{\prime}=L+1}^{\infty} \mathcal{M}_{\ell^{\prime}-L}\left(t-t_{0}\right) \psi_{\ell^{\prime}}\left(t_{0}\right) .
\end{aligned}
$$

The index $\ell$ can now take values in $\mathbb{Z}$ and the unscaled region goes from $\ell_{-}=1$ to $\ell_{+}=L$. In the practical numerical implementation, the $\mathrm{BH}$ chain has to be sufficiently long in order to absorb the outgoing flux. The last two lines of Eq. (27) still contain the propagation of the initial population of the leads into the scattering region, which is unaffected by the SECS transformation.

\section{Results}

We now compare the SECS and TBC methods with each other and with the well established method of complex absorbing potentials (CAPs). The imaginary part of such a complex potential renders the Hamiltonian non-Hermitian and thus the evolution non-unitary. For 
the sake of comparability with the SECS method, we choose the absorbing potential

$V_{\ell}^{\mathrm{CAP}}=-i \operatorname{Im}\left(q_{\ell}\right)$,

with $q_{\ell}$ being defined, as in Sec. 4, by the discretization of Eq. (23).

The TBC, SECS, and CAP methods are applied to the case of free kinetic propagation along a homogeneous and noninteracting $\mathrm{BH}$ chain, as well as to the case of propagation across a symmetric double-barrier configuration in the presence of interaction, which can be seen as an atomic quantum dot. At the initial time $t_{0}$, the $\mathrm{BH}$ chain is considered to be either completely empty (i.e. $\psi_{\ell}\left(t_{0}\right)=0$ for all $\ell \in \mathbb{Z}$ ) or populated with some randomly selected complex amplitudes on each site. The coupling to the source is ramped according to

$\kappa(t)=\frac{1}{1+e^{-\left(J\left(t-t_{0}\right)-50\right) / 5}}$.

To solve the differential equations, we use a RungeKutta Fehlberg (RKF) method. This method is of order $\mathcal{O}\left(\delta t^{4}\right)$ with an error estimator of order $\mathcal{O}\left(\delta t^{5}\right)$, which allows one to adapt the numerical time step $\delta t$ in order to keep the numerical solution as close as possible to the mathematically true solution of the equations.

\subsection{Free case with empty leads}

We first consider the case of a free kinetic propagation, i.e. $V_{\ell}=g_{\ell}=0$ for all $\ell \in \mathbb{Z}$, and compare the density profiles obtained by the TBC, SECS, and CAP methods against the expected value for the stationary density $n^{\varnothing}$. The latter can be analytically calculated with Eq. (16) by restricting the central region $\mathcal{Q}$ to a single site. We obtain

$n^{\varnothing}=\lim _{t \rightarrow \infty}|\psi(t)|^{2}=\lim _{t \rightarrow \infty} \frac{\mathcal{N}|\kappa(t)|^{2}}{4 J^{2}-\mu^{2}}$.

In Fig. 2, we represent the propagation of free particles in a BH chain consisiting of 100 sites within the $\mathcal{Q}$ region. The source is located at the first site in this region. The $\mathcal{L}$ and $\mathcal{R}$ regions are treated according to TBC, CAP or SECS. We chose the smoothing parameter $\lambda=0.1$ and the maximal scaling angle $\theta=1.5$. We can see that the three methods agree with each other and reproduce the analytical value (30) of the on-site density within the $\mathcal{Q}$ region. Moreover, the three methods seem to be stable for long propagation times, which allows us to study the steady state of this scattering process with confidence. For a total propagation time of $J t=250$, the SECS method is found to be slightly faster than the CAP method. We believe that this is

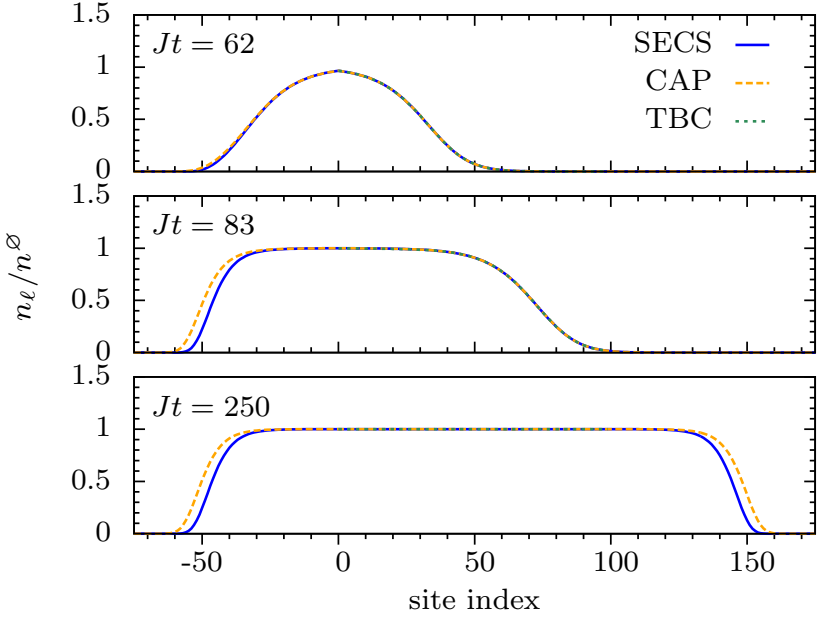

Fig. 2 (color online) Density profiles for the case of free propagation (i.e. $g_{\ell}=V_{\ell}=0$ ) in a homogeneous $\mathrm{BH}$ chain for three different propagation times $t$. The source of atoms is connected at site $\ell_{S}=1$. The TBC method (red solid line), the SECS method (green dashed line), as well as the CAP method (blue dotted line) yield practically identical densities within the region $\mathcal{Q}$ defined from site 1 to site 100, which agree there with the analytical expression (30) for the stationary density. Differences between the numerical methods naturally appear within the leads (in which the results obtained by the TBC method are not displayed). While SECS is found to absorb the outgoing flux most efficiently in terms of computation time, TBC is the slowest method because of the integral in the decay term in Eq. (16).

due to CAP absorbing the outgoing flux less efficiently than SECS; hence, more sites in the leads contribute to the error computed by the adaptive RKF method and consequently more time steps have to be taken in order to reach the final time. On the other hand, the TBC method, while being exact from the formal point of view, is substantially slower (about 1000 times for this particular comparison) than the other two methods. This can be explained by the fact that the numerical evaluation of the integral in the decay term is very costly and and has to be re-done at any individual time step since this integral is a convolution of the memory kernel with the local history of the wavefunction.

\subsection{Free case with populated leads}

Let us now study the influence of nonvanishing initial populations in the leads. We generate the initial condition on the site $\ell$ according to

$\psi_{\ell}\left(t=t_{0}\right)=\frac{1}{2}\left(\mathcal{A}_{\ell}+i \mathcal{B}_{\ell}\right)$

where $\mathcal{A}_{\ell}$ and $\mathcal{B}_{\ell}$ are real, independent Gaussian random variables with unit variance and zero mean, such 


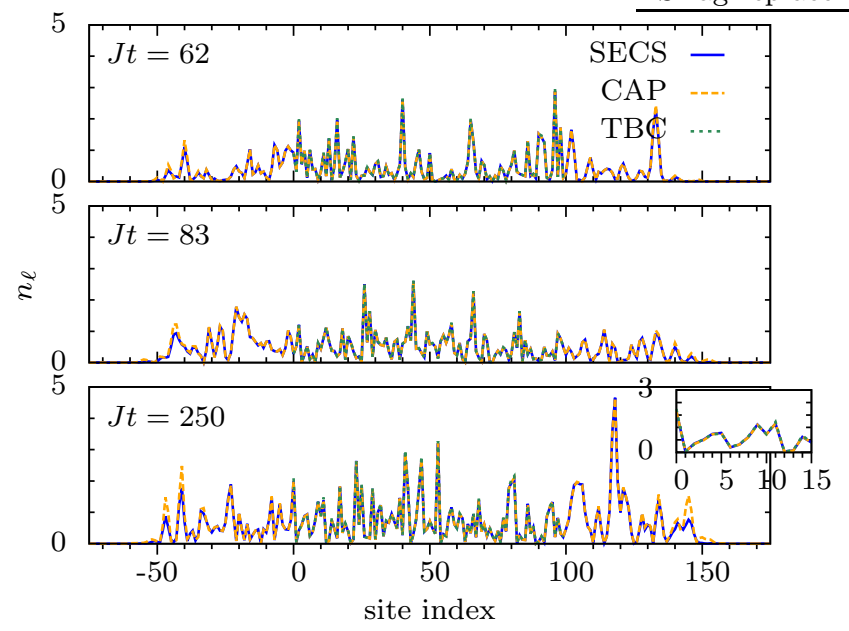

Fig. 3 (color online) Density profiles for free propagation (i.e. $g_{\ell}=V_{\ell}=0$ ) with random initial populations in the leads of the BH chain. There is no source of atoms in this calculation. As is clearly visible in the inset, all three methods compare very well in the central region $\mathcal{Q}$ which is again defined from site 1 to site 100. SECS absorbs the outgoing flux more effectively in the leads than CAP and appears to be the most efficient method in terms of computation time.

that

$$
\begin{aligned}
\left\langle\mathcal{A}_{\ell}\right\rangle & =\left\langle\mathcal{B}_{\ell}\right\rangle=0, \\
\left\langle\mathcal{A}_{\ell^{\prime}} \mathcal{A}_{\ell}\right\rangle & =\left\langle\mathcal{B}_{\ell^{\prime}} \mathcal{B}_{\ell}\right\rangle=\delta_{\ell^{\prime}, \ell}, \\
\left\langle\mathcal{A}_{\ell^{\prime}} \mathcal{B}_{\ell}\right\rangle & =0
\end{aligned}
$$

for all $\ell, \ell^{\prime} \in \mathbb{Z}$. As we shall point out in a forthcoming paper [49, such initial conditions arise when applying the truncated Wigner method to the transport of BoseEinstein condensates in the context of atom lasers.

We consider again a homogeneous non-interacting $\mathrm{BH}$ chain and used the same parameters as in Sec. 5.1 for SECS and CAP. Owing to the linearity of the time evolution in the case $g_{\ell}=0$ for all $\ell \in \mathbb{Z}$, we can, without loss of generality, set the coupling to the source to zero, $\kappa(t)=0$, for all times $t$ and study the evolution of the random initial populations within an isolated waveguide (since the effect of the source was already investigated in Sec. 5.1). The results of this simulation are shown in Fig. 3. We arrive at the same conclusions as in Sec. 5.1 All three methods agree with each other and yield nearly identical on-site densities. In particular, there is no artificial accumulation of the total population within the central $\mathcal{Q}$ region due to an inefficient absorption of the outgoing flux at the boundaries. Again, the computational effort for SECS is appreciably lower than for CAP and substantially lower than for the integro-differential TBC method.

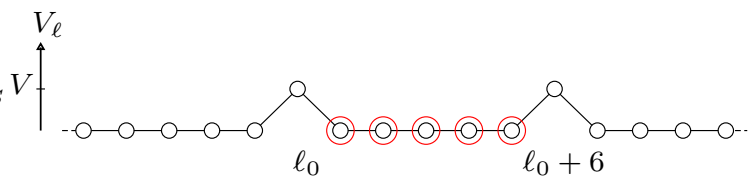

Fig. 4 (color online) One dimensional chain for the quantum dot model (see Eq. 33). Plotted is the on-site potential $V_{\ell}$ as a function of the site index $\ell$. The red circles represent sites where the atoms can interact.

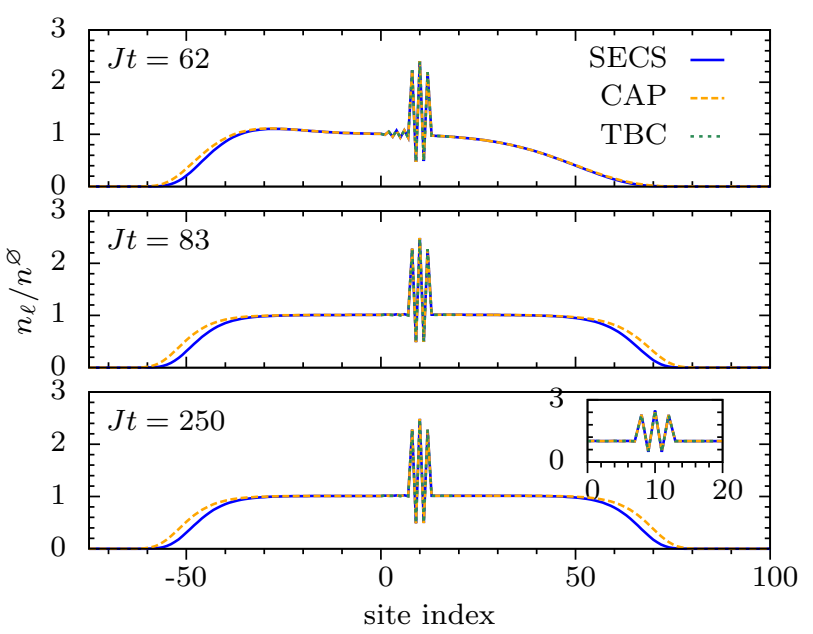

Fig. 5 (color online) Density profiles for a resonant propagation across a quantum dot in the $\mathrm{BH}$ chain, defined in Eqs. (33) and (34), with $\mu=-0.242 J$ and $g=0.1 J$ before (upper panel) and after (middle and lower panels) reaching the stationary state. The source of atoms is located at site 1 . As shown in the inset, all three methods compare very well in the central region $\mathcal{Q}$ defined from site 1 to site 20 .

\subsection{Quantum dot}

In this section we study the effects of a nonvanishing potential and a finite on-site interaction on the scattering process. As displayed in Fig. 4, we specifically consider a double-barrier configuration defined by

$V_{\ell}=V\left(\delta_{\ell, \ell_{0}}+\delta_{\ell, \ell_{0}+6}\right)$,

which can be seen as an atomic quantum dot. Interaction is present only within the dot, i.e. we define

$g_{\ell}=g \sum_{j=1}^{5} \delta_{\ell, \ell_{0}+j}$

and choose $g=0.1 J$.

Figure 5 shows the density profiles obtained by the TBC, SECS, and CAP methods for the chemical potential $\mu=-0.242 J$ which corresponds to a resonance of the double-barrier configuration at the interaction strength $g=0.1 J$. Again, the three methods yield nearly identical results, which confirms their validity. This does not change if we add, as in Sec. [5.2, nonvanishing initial populations in the leads. Finally, Fig. 6 shows the 


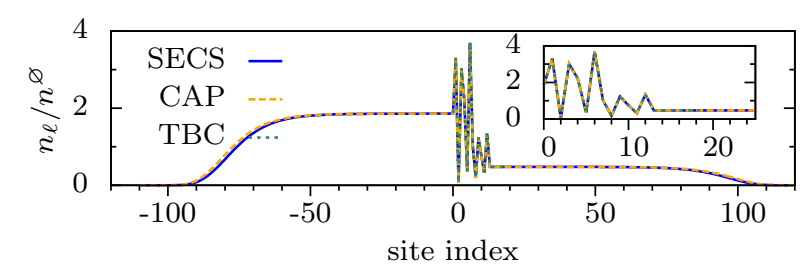

Fig. 6 (color online) Same as Fig. 5 for $J t=250$ and the chemical potential $\mu=-0.8 J$ which at $g=0.1 J$ gives rise to non-resonant transport with finite reflection. Again, very good agreement between all three methods is found.

case of imperfect transmission at the chemical potential $\mu=-0.8 J$. As in the noninteracting cases studied in the previous subsections, we find that the SECS method is more efficient (i.e. less time consuming) than the CAP and TBC methods.

\section{Conclusions}

In summary, we introduced in this work the method of smooth exterior complex scaling (SECS) to the meanfield description of the one-dimensional transport of Bose-Einstein condensates within a guided atom laser configuration. While this method is formally exact in a continuous system, imprecisions necessarily arise if the space is discretized in the framework of a finitedifference representation of the Gross-Pitaevskii equation. We showed how to avoid this problem by choosing a sufficiently large smoothing parameter in the implementation of SECS. A comparison with the (numerically inefficient) introduction of perfectly transparent boundary conditions, which are obtained from an analytical elimination of the semi-infinite leads of the waveguide (assuming that spatial inhomogeneities and nonvanishing interactions are restricted to a finite scattering region within the waveguide), yields very good quantitative agreement. This was specifically tested for the case of resonant and non-resonant transport through an atomic quantum dot configuration consisting of a sequence of two symmetric barriers within which the interaction was assumed to be finite. We furthermore showed that the SECS method is appreciably more efficient than the method of complex absorbing potentials that are defined with a comparable smoothing parameter.

In contrast to the method of absorbing boundary conditions proposed in Ref. 12, which are adapted to outgoing waves with relatively well-defined wave numbers, the SECS method can also account for the presence of density fluctuations that arise from finite random initial populations within the waveguide of the atom laser. This implies that SECS can be applied in the framework of the Truncated Wigner method [50] which approximately accounts for the effect of quantum fluctuations beyond the mean-field description of the propagating condensate. This specific application, as well as the use of SECS within the many-body matrix product state (MPS) algorithm [51 55], shall be discussed in a forthcoming publication [49].

\section{Acknowledgements}

We acknowledge financial support from Fonds de la Recherche Scientifique de Belgique (F.R.S.-FNRS) and the DFG Forschergruppe FOR760" Scattering Systems with Complex Dynamics". The computational resources have been provided by the Consortium des Équipements de Calcul Intensif (CÉCI), funded by the F.R.S.-FNRS under Grant No. 2.5020.11.

\section{References}

1. A. Micheli, A. J. Daley, D. Jaksch, and P. Zoller, Phys. Rev. Lett. 93, 140408 (2004).

2. B. T. Seaman, M. Krämer, D. Z. Anderson, and M. J. Holland, Phys. Rev. A 75, 023615 (2007).

3. R. A. Pepino, J. Cooper, D. Z. Anderson, and M. J. Holland, Phys. Rev. Lett. 103, 140405 (2009).

4. J.-P. Brantut, J. Meineke, D. Stadler, S. Krinner, and T. Esslinger, Science 337, 1069 (2012).

5. M. Bruderer and W. Belzig, Phys. Rev. A 85, 013623 (2012).

6. L. H. Kristinsdóttir, O. Karlström, J. Bjerlin, J. C. Cremon, P. Schlagheck, A. Wacker, and S. M. Reimann, Phys. Rev. Lett. 110, 085303 (2013).

7. D. B. Gutman, Y. Gefen, and A. D. Mirlin, Phys. Rev. B 85, 125102 (2012).

8. A. Ivanov, G. Kordas, A. Komnik, and S. Wimberger, Eur. Phys. J. B 86, 345 (2013).

9. W. Guerin, J.-F. Riou, J. Gaebler, V. Josse, P. Bouyer, and A. Aspect, Phys. Rev. Lett. 97, 200402 (2006).

10. A. Couvert, M. Jeppesen, T. Kawalec, G. Reinaudi, R. Mathevet, and D. Guéry-Odelin, Europhys. Lett. 83, 50001 (2008).

11. G. L. Gattobigio, A. Couvert, B. Georgeot, and D. GuéryOdelin, Phys. Rev. Lett. 107, 254104 (2011).

12. T. Shibata, Phys. Rev. B 43, 6760 (1991).

13. T. Paul, K. Richter, and P. Schlagheck, Phys. Rev. Lett. 94, 020404 (2005).

14. T. Paul, M. Hartung, K. Richter, and P. Schlagheck, Phys. Rev. A 76, 063605 (2007).

15. T. Paul, P. Leboeuf, N. Pavloff, K. Richter, and P. Schlagheck, Phys. Rev. A 72, 063621 (2005).

16. T. Ernst, T. Paul, and P. Schlagheck, Phys. Rev. A 81, 013631 (2010).

17. R. Kosloff and D. Kosloff, J. Comput. Phys. 63, 363 (1986).

18. U. V. Riss and H.-D. Meyer, J. Phys. B: At. Mol. Opt. Phys. 31, 2279 (1998).

19. N. Moiseyev, L. D. Carr, B. A. Malomed, and Y. B. Band, J. Phys. B 37, L193 (2004). 
20. K. Rapedius, C. Elsen, D. Witthaut, S. Wimberger, and H. J. Korsch, Phys. Rev. A 82, 063601 (2010).

21. E. Balslev and J. M. Combes, Commun. Math. Phys. 22, 280 (1971).

22. B. Simon, Ann. Math. 97, 247 (1973).

23. W. P. Reinhardt, Annu. Rev. Phys. Chem. 33, 223 (1982).

24. B. Junker, Adv. Atom. Mol. Phys. 18, 207 (1982).

25. Y. Ho, Phys. Rep. 99, 1 (1983).

26. P.-O. Löwdin, Adv. Quant. Chem. 19, 87 (1988).

27. N. Moiseyev, Phys. Rep. 302, 212 (1998).

28. N. Moiseyev and L. S. Cederbaum, Phys. Rev. A 72, 033605 (2005).

29. P. Schlagheck and T. Paul, Phys. Rev. A 73, 023619 (2006).

30. S. Wimberger, P. Schlagheck, and R. Mannella, J. Phys. B 39, 729 (2006).

31. P. Schlagheck and S. Wimberger, Appl. Phys. B 86, 385 (2006).

32. B. Simon, Phys. Lett. A 71, 211 (1979).

33. P. Froelich, B. Jeziorski, W. Kolos, H. Monkhorst, A. Saenz, and K. Szalewicz, Phys. Rev. Lett. 71, 2871 (1993).

34. A. Saenz, Phys. Rev. A 67, 033409 (2003).

35. T. N. Rescigno, Science 286, 2474 (1999).

36. C. W. McCurdy, M. Baertschy, and T. N. Rescigno, J. Phys. B: At. Mol. Opt. Phys. 37, R137 (2004).

37. F. He, C. Ruiz, and A. Becker, Phys. Rev. A 75, 053407 (2007).

38. L. Tao, W. Vanroose, B. Reps, T. N. Rescigno, and C. W. McCurdy, Phys. Rev. A 80, 063419 (2009).

39. L. Tao and A. Scrinzi, New J. Phys. 14, 013021 (2012).

40. A. Scrinzi, New J. Phys. 14, 085008 (2012).

41. A. Scrinzi and N. Elander, J. Chem. Phys. 98, 3866 (1993).

42. A. Scrinzi, Phys. Rev. A 81, 053845 (2010).

43. C. W. McCurdy and F. Martín, J. Phys. B: At. Mol. Opt. Phys. 37, 917 (2004).

44. N. Rom, E. Engdahl, and N. Moiseyev, J. Chem. Phys. 93, 3413 (1990).

45. N. Elander, S. Levin, and E. Yarevsky, Phys. Rev. A 67, 062508 (2003).

46. T. Hartmann, J. Michl, C. Petitjean, T. Wellens, J.-D. Urbina, K. Richter, and P. Schlagheck, Ann. Phys. 327, 1998 (2012).

47. J.-F. Riou, Y. Le Coq, F. Impens, W. Guerin, C. Bordé, A. Aspect, and P. Bouyer, Phys. Rev. A 77, 033630 (2008).

48. D. J. Kalita and A. K. Gupta, J. Chem. Phys. 134, 094301 (2011).

49. J. Dujardin, A. Argüelles, and P. Schlagheck, In preparation.

50. M. Steel, M. Olsen, L. Plimak, P. Drummond, S. Tan, M. Collett, D. Walls, and R. Graham, Phys. Rev. A 58, 4824 (1998)

51. F. Verstraete, D. Porras, and J. Cirac, Phys. Rev. Lett. 93, 227205 (2004).

52. G. Vidal, Phys. Rev. Lett. 91, 147902 (2003).

53. G. Vidal, Phys. Rev. Lett. 93, 040502 (2004). 